\title{
Novel LTBP3 mutations associated with thoracic aortic aneurysms and dissections
}

\author{
Guoyan Zhu' ${ }^{1}$ Mingyao Luo², Qianlong Chen ${ }^{1}$, Yinhui Zhang ${ }^{1}$, Kun Zhao ${ }^{1}$, Yujing Zhang ${ }^{1}$, Chang Shu², \\ Hang Yang ${ }^{1 *}$ and Zhou Zhou ${ }^{1 *}$ (D)
}

\begin{abstract}
Background: Thoracic aortic aneurysm and dissection (TAAD) is a hidden-onset but life-threatening disorder with high clinical variability and genetic heterogeneity. In recent years, an increasing number of genes have been identified to be related to TAAD. However, some genes remain uncertain because of limited case reports and/or functional studies. LTBP3 was such an ambiguous gene that was previously known for dental and skeletal dysplasia and then noted to be associated with TAAD. More research on individuals or families harboring variants in this gene would be helpful to obtain full knowledge of the disease and clarify its association with TAAD.
\end{abstract}

Methods: A total of 266 TAAD probands with no causative mutations in known genes had been performed wholeexome sequencing (WES) to identify potentially pathogenic variants. In this study, rare LTBP3 variants were the focus of analysis.

Results: Two compound heterozygous mutations, c.625dup (p.Leu209fs) and c.1965del (p.Arg656fs), in LTBP3 were identified in a TAAD patient along with short stature and dental problems, which was the first TAAD case with biallelic LTBP3 null mutations in an Asian population. Additionally, several rare heterozygous LTBP3 variants were also detected in other sporadic TAAD patients.

Conclusion: The identification of LTBP3 mutations in TAAD patients in our study provided more clinical evidence to support its association with TAAD, which broadens the gene spectrum of LTBP3. LTBP3 should be considered to be incorporated into the routine genetic analysis of heritable aortopathy, which might help to fully understand its phenotypic spectrum and improve the diagnostic rate of TAAD.

Keywords: Thoracic aortic aneurysm and dissection, LTBP3 gene, Genetic mutation

\section{Background}

Thoracic aortic aneurysms are often asymptomatic, as they progressively enlarge and are finally aware of being diagnosed clinically when dissections or ruptures occur, which are life-threatening and can cause sudden death in

\footnotetext{
*Correspondence: chrisyang_1999@163.com; zhouzhou@fuwaihospital.org 1 State Key Laboratory of Cardiovascular Disease, Beijing Key Laboratory for Molecular Diagnostics of Cardiovascular Diseases, Diagnostic Laboratory Service, Fuwai Hospital, National Center for Cardiovascular Diseases, Chinese Academy of Medical Sciences and Peking Union Medical College, Beijing 100037, China

Full list of author information is available at the end of the article
}

up to $50 \%$ of patients $[1,2]$. Therefore, early diagnosis and timely treatment of thoracic aortic aneurysm and dissection (TAAD) are very important for reducing mortality.

Many factors contribute to TAAD development, while genetic defects play a major role. Marfan syndrome (MFS) is the most well-known heritable aortic disease, and other syndromic connective diseases involving the aorta, such as Loeys-Dietz syndrome (LDS) and vascular Ehlers-Danlos syndrome (EDS), are less commonly seen. An increasing number of causative mutations have also been identified in nonsyndromic TAAD patients, but they can only explain a small proportion (approximately $20 \%$ ) of TAAD original author(s) and the source, provide a link to the Creative Commons licence, and indicate if changes were made. The images or other third party material in this article are included in the article's Creative Commons licence, unless indicated otherwise in a credit line to the material. If material is not included in the article's Creative Commons licence and your intended use is not permitted by statutory regulation or exceeds the permitted use, you will need to obtain permission directly from the copyright holder. To view a copy of this licence, visit http://creativecommons.org/licenses/by/4.0/. The Creative Commons Public Domain Dedication waiver (http://creativeco mmons.org/publicdomain/zero/1.0/) applies to the data made available in this article, unless otherwise stated in a credit line to the data. 
families [3], which suggests that there are many unrecognized genes to be explored.

To date, 11 genes (ACTA2, COL3A1, FBN1, LOX, MYH11, MYLK, PRKG1, SMAD3, TGFB2, TGFBR1, TGFBR2) have been confirmed to be responsible for heritable TAAD [1]. These genes encode proteins involved in smooth muscle cell contraction, the extracellular matrix, and transforming growth factor-beta (TGF- $\beta$ ) signaling [4]. Several genes, such as $B G N$, FOXE3, HCN4, MAT2A, MFAP5, SMAD2, and TGFB3, remain uncertain [1] because they were recently discovered, and there have been few reported cases and insufficient evidence. However, as research continues, their association with TAAD may become clear.

$\angle T B P 3$, which encodes an extracellular matrix protein, is highly expressed in human ovaries, prostate, fat, heart, and skeletal muscle [5, 6]. Mutations in this gene were previously known to be associated with skeletal dysplasia, such as dental anomalies and short stature (DASS; OMIM\#601216), geleophysic dysplasia 3 (GPHYSD3; OMIM\#617809), and acromicric dysplasia (ACMICD; OMIM \#102370) [7-9]. Until in 2018, LTBP3 was firstly reported to predispose individuals to TAAD [10]. Although the underlying mechanisms are not fully elucidated, the involvement of LTBP3 in TAAD is not unexpected considering its close association with $F B N 1$, the MFS gene $[11,12]$.

$L T B P 3$ belongs to the latent TGF- $\beta$ binding protein (LTBP) family and contains several modules including TGF- $\beta$-binding (TB) domain, epidermal growth factor (EGF), 4 Cys, hybrid, and calcium-binding EGF domains [13], which closely resembles that of fibrillin 1. Studies have shown that LTBP3 is assisted by fibrillin-1 to incorporate into the extracellular matrix, which can be hindered in the absence of fibrillin-1 microfibrils in vivo or in vitro [12]. Meanwhile, it has an essential role in regulating the TGF- $\beta$ signaling pathway $[5,6]$. Therefore, LTBP3 might be involved in TAAD. Nevertheless, only a few cases with $L T B P 3$ mutations have been reported and we may not understand the full clinical spectrum of the disease. Aortic deformity might be an important characteristic that occurs more frequently but has been neglected in the past.

In this study, we reported two compound heterozygous LTBP3 mutations in a TAAD patient along with DASS and several rare heterozygous $L T B P 3$ variants in sporadic TAAD patients. Our results provide more clinical evidence supporting that LTBP3 mutations might be responsible for heritable TAAD.

\section{Materials and methods}

\section{Patients}

Patients with TAAD from the Center of Vascular Surgery in Fuwai Hospital were referred to our Diagnostic Laboratory Service for genetic testing. The targeted sequencing contained 15 genes (FBN1, TGFB2, SMAD3, TGFBR1, TGFBR2, ACTA2, MYH11, SMAD4, MYLK, NOTCH1, PRKG1, SKI, COL3A1, SLC2A10, FBN2) associated with aortic disease and was performed as previously reported [14]. Among them, 266 patients who did not have an identified causative mutation in the panel testing were recruited in our study. Wholeexome sequencing was then performed to identify potentially pathogenic mutations.

\section{Whole exome sequencing (WES)}

Genomic DNA was extracted from EDTA-anticoagulated whole blood of patients and their relatives using a QIAamp DNA Blood Mini Kit (Qiagen, Hilden, Germany) according to the manufacturer's instructions [15, 16]. The DNA samples and sequencing data were processed as previously described [17]. WES was performed on an Illumina HiSeq2500 platform (Illumina Inc., San Diego, CA, USA) using the TruSeq Rapid PE Cluster kit V2 or TruSeq Rapid SBS kit V2 - HS (Illumina Inc., San Diego, CA, USA).

\section{Bioinformatics analysis}

The process of WES bioinformatics analysis can be roughly divided into three modules: data preprocessing, variant detection, and annotation. FastP software was used to filter the low-quality sequence reads [18], and sequence reads were aligned to the reference genome hg19 using BWA (Burrows-Wheeler Aligner) MEM software $[15,19,20]$. Picard (MarkDuplicates) software (https:/github.com/broadinstitute/picard) was used to label repetitive reads, and GATK3.7 software was used to correct systematic errors in the sequencing process. The GATK Haplotypecaller (https://gatk.broadinstitute. org) was used in variant calling, and Annovar software (https://annovar.openbioinformatics.org/en/latest/) was used for annotation analysis. High quality variants (mean sequencing depth $>=20 \times$, alternation frequency (AltFeq) between 0.3 and 0.6 or $>0.95$, strand bias (STB) between 0.5 and 0.7 ) detected by WES were filtered for further analysis. Variants based on the $<1 \%$ minor allele frequency (MAF) in any gnomAD (version 2.1) population (gnomAD_popmax) and location in the coding regions and variable splicing sites (canonical \pm 1 or 2 splice sites or splice sites of either dbscSNV_ADA or dbscSNV_RF > 0.6 were retained. Then $\angle T B P 3$ rare variants with a MAF $<0.01$ and $<0.00005$ in gnomAD were focused separately 
Table 1 Compound heterozygous mutations in LTBP3 identified in patient AD2002

\begin{tabular}{|c|c|c|c|c|c|}
\hline Mutations & Inheritance & MAF & Pathogenicity & ACMG evidence & Genotype of relatives \\
\hline $\begin{array}{l}\text { LTBP3: NM_001130144: c.1965del } \\
\text { (p.Arg656Alafs*6) }\end{array}$ & Autosomal recessive & 0 & Pathogenic & PVS1, PM2, PM3 & $\begin{array}{l}\text { Father: -/- } \\
\text { Mother: +/- } \\
\text { Sister: +/- } \\
\text { Elder son: -/- } \\
\text { Youngest son: -/- }\end{array}$ \\
\hline $\begin{array}{l}\text { LTBP3: NM_001130144: c.625dup } \\
\text { (p.Leu209Profs*38) }\end{array}$ & Autosomal recessive & 0 & Pathogenic & PVS1, PS2, PM2, PM3 & $\begin{array}{l}\text { Father:-/- } \\
\text { Mother:-/- } \\
\text { Sister: -/- } \\
\text { Elder son: +/- } \\
\text { Youngest son: +/- }\end{array}$ \\
\hline
\end{tabular}

MAF, minor allele frequence; $-/-$, wild type; $+/-$, heterozygous

for autosomal recessive and dominant conditions. The variant interpretation was then performed according to ACMG guidelines [21, 22]. Variants detected in our study were written according to the HGVS nomenclature rules.

\section{Sanger sequencing}

All the mutations detected by WES were verified by Sanger sequencing. According to the reference genomic sequences of the Human Genome from GenBank in NCBI [22], Primer3 Input(version 0.4.0) was used to design primer pairs. Sanger sequencing was performed with the following primers:

\begin{tabular}{lll}
\hline Gene & Forward primer & Reverse primer \\
\hline LTBP3_Exon1 & CGGCCCTCTACTCCCTTC & GTCCGCTTGCAGATCACC \\
LTBP3_Exon2 & GAGGAGGGGAAAGAG & GGCGTTCGAGCTCTCAAT \\
& ACAGG & \\
LTBP3_EXon8 & CACCGGTGAGTCAGG & TTGGGGGTTAGACTGTGA \\
& GTTAC & GG \\
LTBP3_Exon10 & ACTTCATGGCCCCATCTT & CCCAGTGATTTAGCCCTTGA \\
& CT & \\
LTBP3_EXon13 & CTTGGCCTACCCGTT & AGTGACCGGGAAAGTTGA \\
& CTTCT & TG \\
\hline
\end{tabular}

\section{Results}

Homozygous or compound heterozygous LTBP3 mutations were first reviewed in our 266 WES data, with a $\mathrm{MAF}<0.01$ in gnomAD. Two heterozygous mutations, c.625dup (p.Leu209fs) and c.1965del (p.Arg656fs), were detected in patient AD2002. These two variants were not recorded in the population database, and both of them were predicted to undergo nonsense-mediated decay. They were both confirmed by Sanger sequencing. After detecting his parents' genotype, we found that one of them (c.1965del, p.Arg656fs) was inherited from his mother, while the other (c.625dup, p.Leu209fs) was de novo. To determine whether they were in trans or cis, the genotypes of their offspring were also detected. His twin sons carried only the variant c.625dup, which highly suggested that these two variants were on opposite chromosomes. Therefore, these compound heterozygous variants could be interpreted as pathogenic according to the ACMG guidelines (Table 1) and were causative for his disease.

Patient AD2002 was a 42-year-old man, $165 \mathrm{~cm} / 63$ $\mathrm{kg}$, with no hypertension history. He had sudden back pain during a business trip and went to the local hospital. Echocardiography showed dilatation of the aortic sinus $(53 \mathrm{~mm})$ and ascending aorta $(38 \mathrm{~mm})$, and thoracic computed tomography $(\mathrm{CT})$ revealed aortic dissection in the descending aorta (DeBakey type III). TEVAR surgery was then performed. Two months later, he came to Fuwai Hospital for reexamination and a genetic test. After recognizing his two LTBP3 mutations, we further asked about his symptoms and family history. He also had spinal stenosis and dental anomalies, for which his teeth were all replaced at the age of 38. Nevertheless, his mother and sister, as well as his two sons, who all harbored only one heterozygous variant, had no abnormalities (Fig. 1A). No other homozygous or compound heterozygous mutations in this gene were detected in our cohort.

Subsequently, rare heterozygous LTBP3 mutations were filtered in our dataset, with a MAF $<0.00005$ in gno$\mathrm{mAD}$. A total of four missense mutations were detected in four individuals affected with early-onset thoracic aortic dissection (Table 2). None of these patients had significant systemic abnormalities other than the aortic

(See figure on next page.)

Fig. 1 The pedigree and detection of mutations in AD2002. A The pedigree of AD2002 showed that his healthy father (I:1) did not carry mutations, and his mother (I:2) and sister (II:2) were both heterozygous for an LTBP3 variant, C.1965del (p.Arg656Alafs*6), and did not show abnormal clinical characteristics. His healthy twin sons (III:1, III:2) both harbored the heterozygous variant c.625dup (p.Leu209Profs*38). B Chromatograms of Sanger sequencing of AD2002 and his family members. The mutations were all verified by Sanger sequencing 
A

II

III

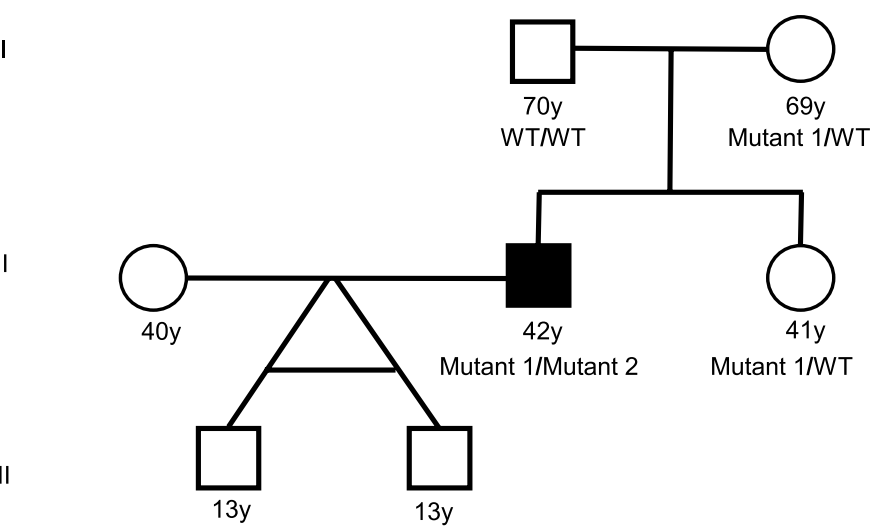

WT/Mutant 2 WT/Mutant 2

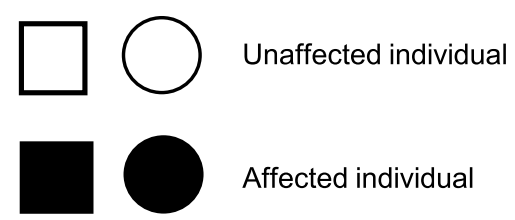

WT Wild Type

Mutant 1 c.1965del (p.Arg656Alafs ${ }^{*} 6$ )

Mutant 2 c.625dup(p.Leu209Profs`38)

B

c.625dup(p.Leu209Profs ${ }^{\star 38)}$
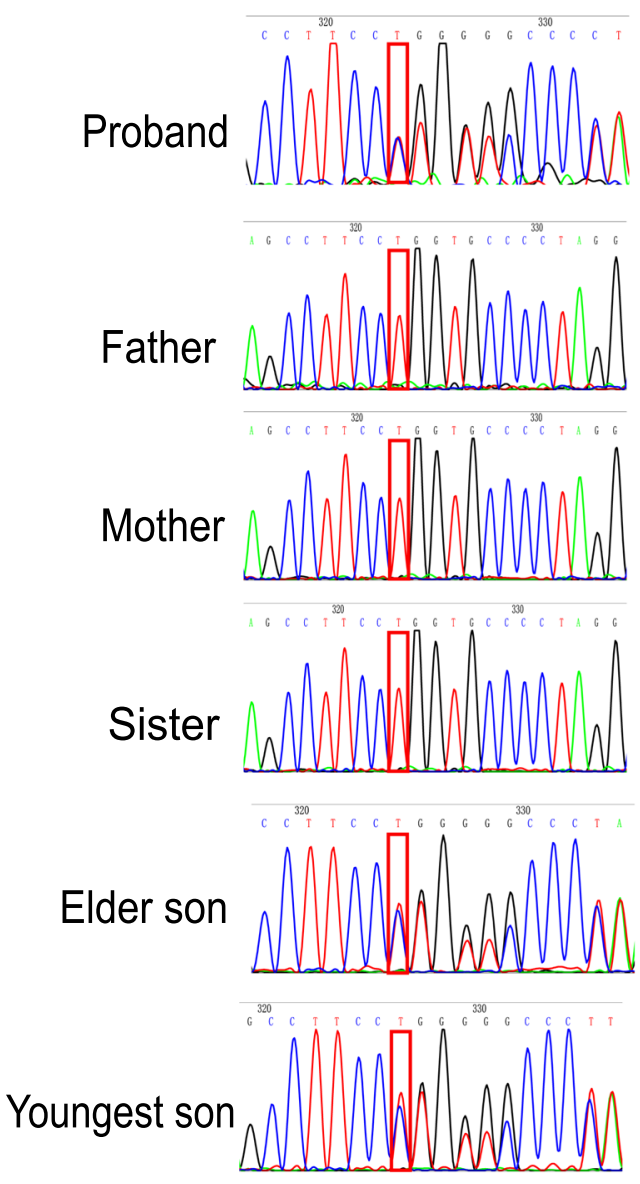

c.1965del(p.Arg656Alafs*6)
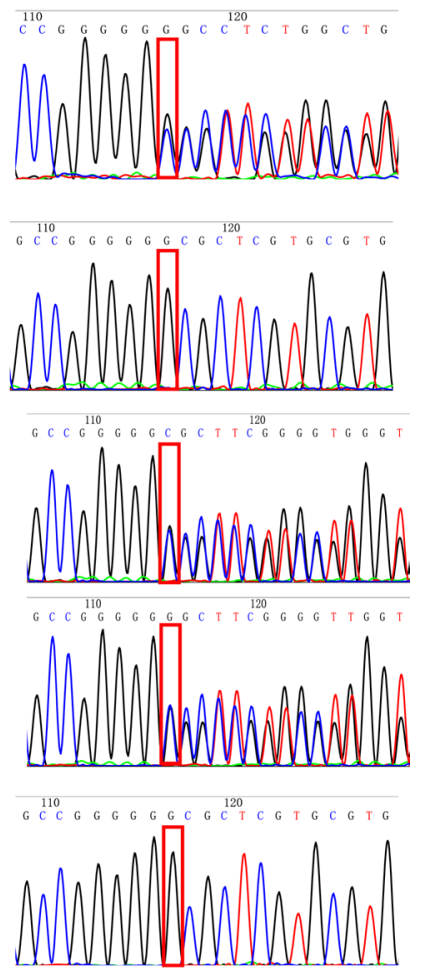

G C C C G G G G G G G C G C T C C G

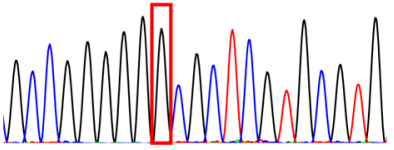

Fig. 1 (See legend on previous page.) 
Table 2 Phenotypic characteristics in sporadic patients with rare heterozygous LTBP3 variants in this study

\begin{tabular}{|c|c|c|c|c|c|c|}
\hline Patient ID & $\begin{array}{l}\text { Age } \\
\text { (year)/ } \\
\text { gender }\end{array}$ & $\begin{array}{l}\text { Height }(\mathrm{cm}) / \\
\text { body weight } \\
(\mathrm{kg})\end{array}$ & Aortic disease & LTBP3 mutations & Other clinical features & $\begin{array}{l}\text { Variants in other HTAAD } \\
\text { gene in ClinGen }\end{array}$ \\
\hline AD721 & $52 / \mathrm{M}$ & $172 / 75$ & Type B aortic dissection & c.1456G > A(p.Gly486Arg) & $\begin{array}{l}\text { Hypertension; Chest pain; } \\
\text { Carotid atherosclerotic } \\
\text { plaque }\end{array}$ & No \\
\hline AD820 & $41 / M$ & $171 / 80$ & Type A aortic dissection & c.1588 A>G(p.Thr530Ala) & $\begin{array}{l}\text { Hypertension; Chest } \\
\text { pain; Aortic regurgitation; } \\
\text { Diabetes }\end{array}$ & $\begin{array}{l}\text { FBN1, c.994 C>T(p.Arg- } \\
\text { 332Cys) }\end{array}$ \\
\hline AD977 & $48 / \mathrm{M}$ & $170 / 80$ & Type B aortic dissection & c.1510G>A(p.Glu504Lys) & $\begin{array}{l}\text { Hypertension; Chest pain; } \\
\text { Aortic regurgitation }\end{array}$ & No \\
\hline AD2076 & $33 / \mathrm{M}$ & $173 / 95$ & $\begin{array}{l}\text { Ascending aortic aneu- } \\
\text { rysm and dissection }\end{array}$ & c.152 C>G(p.Ala51Gly) & Myopia & No \\
\hline
\end{tabular}

LTBP3, NM_001130144; FBN1, NM_000138

disease (details in Table 2). None of the mutations were found in TAAD genes that had been approved by ClinGen in these patients, except for a variant of unknown significance (VUS) in the FBN1 gene. Patient AD820 harbored the $F B N 1$ variant c.994C $>\mathrm{T}$ (p.Arg332Cys) and the LTBP3 variant c.1588A $>\mathrm{G}$ (p.Thr530Ala), both of which remained uncertain significance.

\section{Discussion}

Disturbed TGF- $\beta$ signaling has been extensively implicated in thoracic aortic aneurysm and dissection. Mutations in FBN1 lead to defective ECM microfibrils and dysregulation of TGF- $\beta$ signaling, both of which play a vital role in the development of aortopathy in MFS patients [23-27]. Several genes in the TGF- $\beta$ signaling pathway, such as TGFBR1, TGFBR2, SMAD2, TGFB2, $T G F B 2$, and $S M A D 2$, have been proven to be causative for syndromic TAAD $[28,29]$. The LTBP3 gene encodes latent transforming growth factor-beta binding protein 3, which has a similar structure to FBN1. Both of them have multiple EGF-like repeats and unique 8-cys domains that are interspersed with TB domains [30]. LTBP3 regulates TGF- $\beta$ activity by enabling its secretion, directing it to specific sites in the ECM, and participating in its activation [31-34]. It is widely expressed, including in the skeleton, tooth, heart, and aorta, which suggests that mutations in this gene may lead to a broad range of phenotypes.

Ltbp3-deficient mice were initially documented to have small body sizes, unique craniofacial malformations, bone abnormalities, and dental anomalies [3539], which were consistent with the clinical features in individuals with $L T B P 3$ mutations, recessive-pattern DASS, and dominant-pattern ACMCID and GPHYSD3 patients. In earlier reports, cardiovascular deformities were not described in either mouse model or patient. Subsequently, Zilberberg et al. [34] have shown that lack of Ltbp3 could attenuate the aneurysmal phenotype and prevent premature death of $F b n 1^{\mathrm{mgR} / \mathrm{mgR}}$ mice, probably by the reduced activation of TGF- $\beta$ signaling. However, Guo et al. [10] pointed out that when aortic diameters were normalized by body mass, the diameter of the aortic root and ascending part in $L t b p 3^{-/-} F b n 1^{\mathrm{mgR} / \mathrm{mgR}}$ mice was similar to that in the $F b n 1^{\mathrm{mgR} / \mathrm{mgR}}$ group, and $L t b p 3$ deficiency resulted in spontaneous aortic dilation. These data suggested that $L t b p 3$ was associated with the formation and progression of thoracic aortic aneurysms, although the exact effect and mechanism were not well understood. It was reasonably assumed that $L T B P 3$ mutations might contribute to TAAD by disturbing the TGF- $\beta$ signaling pathway and extracellular matrix assembly. More studies are needed to determine its specific role in TAAD.

Regarding the clinical data, there were only a few reports supporting the role of $L T B P 3$ in the cardiovascular system. Dugan et al. reported that two sisters in a single family with a homozygous truncated mutation of LTBP3 both had mitral valve prolapse (MVP) [7]. Guo et al. reported that biallelic null mutations in LTBP3 could predispose individuals to thoracic aortic aneurysms and dissections, and heterozygous rare $L T B P 3$ variants might be related to an early onset risk of acute aortic dissection [10]. This was the first and to date, the only report that supported $L T B P 3$ mutations that might be responsible for TAAD. Our study identified two compound heterozygous variants in $L T B P 3$ in an aortic dissection patient, along with short stature and dental problems. They were both frameshift mutations, which were predicted to result in nonsense-mediated mRNA decay (NMD). This was consistent with the inherited pattern and mutation types in DASS, and it offered more clinical evidence showing that LTBP3 mutations could cause TAAD. 


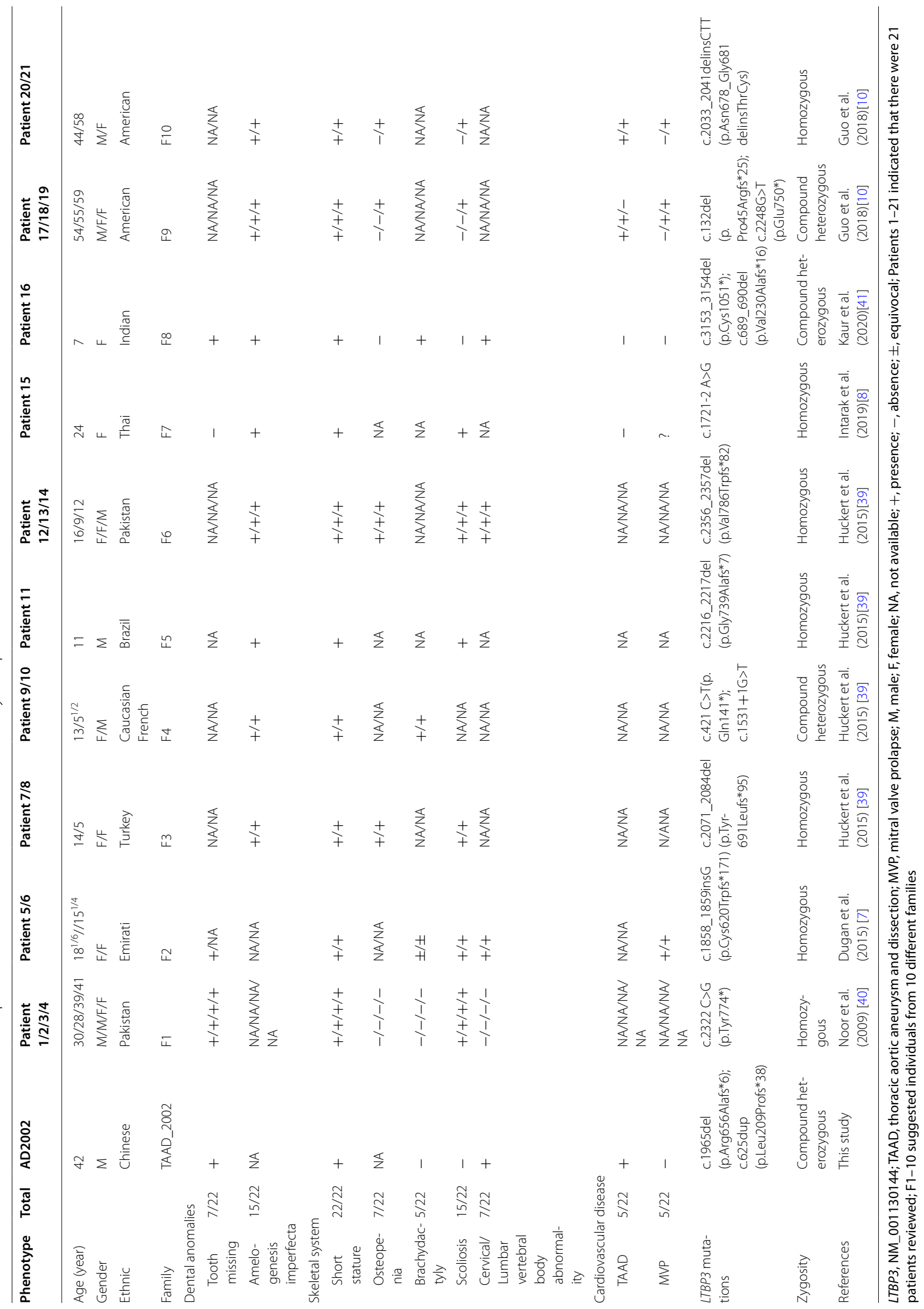




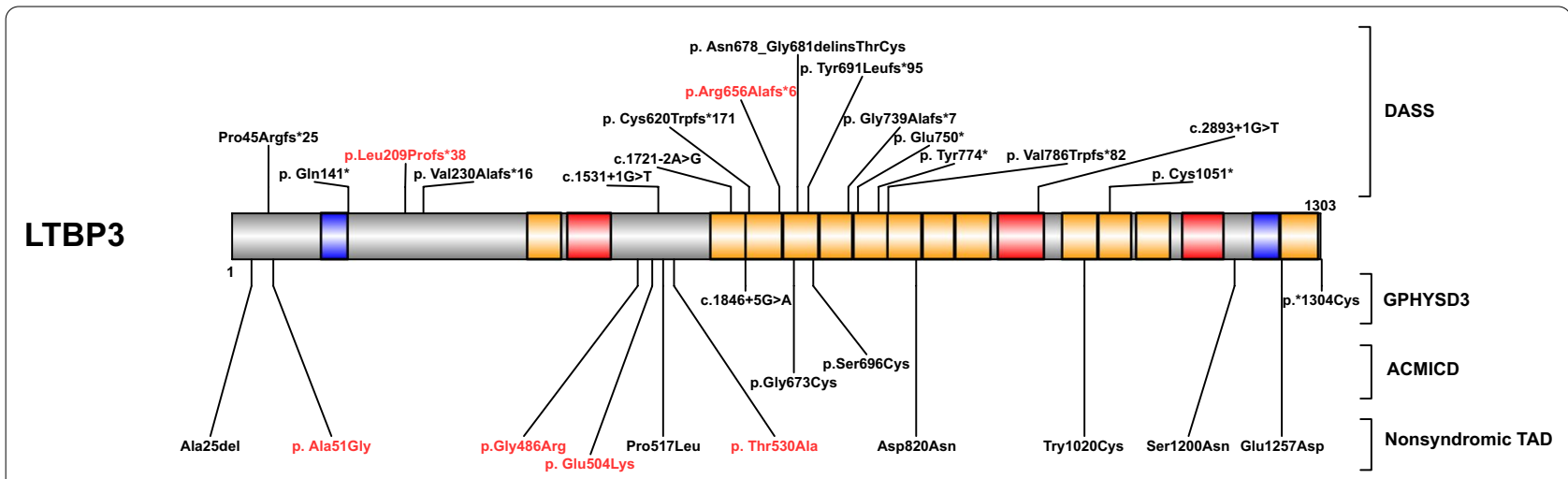

EGF-like domain

EGF-like calcium-binding domain

TB domain

Fig. 2 Schematic diagram of the LTBP3 domain structure and mutation. The identified mutations were indicated above (DASS) and under (GPHYSD3, ACMICD, nonsyndromic TAD) the protein diagram. The mutations identified in this study were shown in red letters, while mutations reported by others were marked in black letters

When reviewing the previously reported cases with homozygous or compound heterozygous LTBP3 null mutations, it was not hard to find that the limited numbers of affected individuals were all diagnosed with DASS at an early age (Table 3). Cardiovascular manifestations were not observed and mentioned specifically. Therefore, we could not exclude the possibility that they might have aortic deformities for the rest of their life. It was reasonable and advisable to follow up these patients to see whether they would have aortic or valvular problems. As LTBP3 was not included in the reevaluation list of the ClinGen expert group on familial TAAD genes [1], it was not incorporated into routine targeted genetic analysis and was mostly not taken into account as a key candidate even in WES. Thus, there was a need to reanalyze the existing WES data on TAAD patients, which might reveal more novel mutations in this gene. More data are needed to assess the frequency of aortic or valvular abnormalities in DASS, which might be more frequent than we thought.

A comprehensive summary of all reported LTBP3 mutations is demonstrated in Fig. 2. It was observed that the majority of bi-allelic loss-of-function LTBP3 mutations, which contributed to DASS, were present in the highly conserved EGF-like calcium-binding domain, while most of the rare monoallelic variants associated ACMICD, GPHYSD3, and thoracic aortic dissection (TAD) were missense mutations located in the same region, indicating that different inheritance modes and natures of $L T B P 3$ might lead to different diseases. Nevertheless, currently reported monoallelic variants in this gene are so limited that further investigations are needed to assess their pathogenicity and apply thorough evaluations to the patients.
The relationship between rare $L T B P 3$ heterozygous variants and aortic dissection risk also remains unclear. Most of the rare $L T B P 3$ variants detected in early-onset of aortic dissection patients by Guo et al. [10] were in the key EGF-like calcium-binding domains, while ours were not, which were less likely to be causative. However, it could not be ruled out that these variants had a mild effect on the development and/or severity of the disease, due to their high CADD predictive scores. Patient AD820 was identified with a variant with unknown significance (PM1, PP3) in the FBN1 gene, c. 994C > T (p.Arg332Cys), which was reported at a low frequency $(0.000008808)$ in the gnomAD database. At the same time, he was identified to carry an $L T B P 3$ heterozygous variant. There was a possibility that these rare variants contributed together to the disease. More cases are needed to fully describe the disease map and genotype-phenotypic association.

\section{Conclusions}

In summary, we identified the first case of a TAAD patient with bi-allelic $L T B P 3$ frameshift mutations in an Asian population, as well as several rare LTBP3 variants in affected individuals with early aortic dissection. This expanded the gene spectrum of LTBP3 and provided more support for its role in TAAD. Our data show the necessity of incorporating this gene into the routine genetic analysis of aortic aneurysms and dissections, which would help to fully understand its phenotypic spectrum and raise the diagnosis rate of TAAD. 


\section{Abbreviations}

TAAD: Thoracic aortic aneurysm and dissection; WES: Whole exome sequencing; DASS: Dental anomalies and short stature; ACMG: American College of Medical Genetics.

\section{Acknowledgements}

We thank all subjects who participated in this study.

\section{Authors' contributions}

GYZ was responsible for data analysis and drafted the manuscript. MYL contributed to recruiting patients and collecting clinical information. QLC contributed to follow-up visit of patients. YHZ and $\mathrm{KZ}$ contributed to the NGS sequencing experiments and Sanger validation. YJZ contributed to bioinformatics analysis. CS contributed to the clinical evaluation and sample management. HY and ZZ designed the study and revised the manuscript. All of the authors have read and approved the final manuscript.

\section{Funding}

This work was supported by the grant of CAMS Initiative for Innovative Medicine, China (No. 2016-12M-1-016).

\section{Availability of data and materials}

All data generated or analysed during this study are included in this published article.

\section{Declarations}

\section{Ethics approval and consent to participate}

This study was approved by the ethics committee of the institutional review board at Fuwai Hospital (Approval No. 2017-877). Informed consent forms were signed by all patients and their relatives who received the genetic tests.

\section{Consent for publication}

All patients gave informed consent to the publication of this study.

\section{Competing interests}

The authors declare that they have no competing interests.

\section{Author details}

${ }^{1}$ State Key Laboratory of Cardiovascular Disease, Beijing Key Laboratory for Molecular Diagnostics of Cardiovascular Diseases, Diagnostic Laboratory Service, Fuwai Hospital, National Center for Cardiovascular Diseases, Chinese Academy of Medical Sciences and Peking Union Medical College, Beijing 100037, China. ${ }^{2}$ State Key Laboratory of Cardiovascular Disease, Center of Vascular Surgery, Fuwai Hospital, National Center for Cardiovascula Diseases, Chinese Academy of Medical Sciences and Peking Union Medical College, Beijing 100037, China.

Received: 17 June 2021 Accepted: 28 November 2021 Published online: 14 December 2021

\section{References}

1. Renard M, Francis C, Ghosh R, Scott AF, Witmer PD, Adès LC, et al. Clinical validity of genes for heritable thoracic aortic aneurysm and dissection. J Am Coll Cardiol. 2018;72:605-15.

2. Puranik R, Chow CK, Duflou JA, Kilborn MJ, McGuire MA. Sudden death in the young. Heart Rhythm. 2005;2:1277-82.

3. Guo D-C, Papke CL, He R, Milewicz DM. Pathogenesis of thoracic and abdominal aortic aneurysms. Ann N Y Acad Sci. 2006;1085:339-52.

4. Zhang $\mathrm{L}$, Wang $\mathrm{H}-\mathrm{H}$. The genetics and pathogenesis of thoracic aortic aneurysm disorder and dissections: the genetics of TAADs. Clin Genet. 2016:89:639-46

5. Penttinen C, Saharinen J, Weikkolainen K, Hyytiäinen M, Keski-Oja J. Secretion of human latent TGF-beta-binding protein-3 (LTBP-3) is dependent on co-expression of TGF-beta. J Cell Sci. 2002;115:3457-68.

6. Saharinen J, Hyytiäinen M, Taipale J, Keski-Oja J. Latent transforming growth factor-beta binding proteins (LTBPS)--structural extracellular matrix proteins for targeting TGF-beta action. Cytokine Growth Factor Rev. 1999;10:99-117.

7. Dugan SL, Temme RT, Olson RA, Mikhailov A, Law R, Mahmood H, et al. New recessive truncating mutation in LTBP3 in a family with oligodontia, short stature, and mitral valve prolapse. Am J Med Genet. 2015;167:1396-9.

8. Intarak N, Theerapanon T, Thaweesapphithak S, Suphapeetiporn K, Porntaveetus T, Shotelersuk V. Genotype-phenotype correlation and expansion of orodental anomalies in LTBP3-related disorders. Mol Genet Genom. 2019;294:773-87.

9. Mclnerney-Leo AM, Le Goff C, Leo PJ, Kenna TJ, Keith P, Harris JE, et al. Mutations in LTBP3 cause acromicric dysplasia and geleophysic dysplasia. Jed Genet. 2016;53:457-64.

10. Guo D, Regalado ES, Pinard A, Chen J, Lee K, Rigelsky C, et al. LTBP3 pathogenic variants predispose individuals to thoracic aortic aneurysms and dissections. Am J Hum Genet. 2018;102:706-12.

11. Yin W, Smiley E, Germiller J, Mecham RP, Florer JB, Wenstrup RJ, et al. Isolation of a novel latent transforming growth factor-beta binding protein gene (LTBP-3). J Biol Chem. 1995;270:10147-60.

12. Zilberberg L, Todorovic V, Dabovic B, Horiguchi M, Couroussé T, Sakai LY, et al. Specificity of latent TGF- $\beta$ binding protein (LTBP) incorporation into matrix: role of fibrillins and fibronectin. J Cell Physiol. 2012;227:3828-36.

13. Robertson IB, Horiguchi M, Zilberberg L, Dabovic B, Hadjiolova K, Rifkin DB. Latent TGF- $\beta$-binding proteins. Matrix Biol. 2015;47:44-53.

14. Yang H, Luo M, Fu Y, Cao Y, Yin K, Li W, et al. Genetic testing of 248 Chinese aortopathy patients using a panel assay. Sci Rep. 2016;6:33002.

15. Zheng $Y, X u$ J, Liang S, Lin D, Banerjee S. Whole exome sequencing identified a novel heterozygous mutation in HMBS gene in a Chinese patient with acute intermittent porphyria with rare type of mild anemia. Front Genet. 2018;9:129.

16. Han P, Wei G, Cai K, Xiang X, Deng WP, Li YB, et al. Identification and functional characterization of mutations in LPL gene causing severe hypertriglyceridaemia and acute pancreatitis. J Cell Mol Med. 2020;24:1286-1299.

17. Chen W, Li W, Ma Y, Zhang Y, Han B, Liu X, et al. Secondary findings in 421 whole exome-sequenced Chinese children. Hum Genom. 2018;12:42.

18. Chen S, Zhou Y, Chen Y, Gu J. fastp: an ultra-fast all-in-one FASTQ preprocessor. Bioinformatics. 2018;34:i884-i890.

19. Li H, Durbin R. Fast and accurate short read alignment with BurrowsWheeler transform. Bioinformatics. 2009;25:1754-60.

20. Dai Y, Liang S, Dong X, Zhao Y, Ren H, Guan Y, et al. Whole exome sequencing identified a novel DAG1 mutation in a patient with rare, mild and late age of onset muscular dystrophy-dystroglycanopathy. J Cell Mol Med.2019;23:811-818.

21. Richards S, Aziz N, Bale S, Bick D, Das S, Gastier-Foster J, et al. Standards and guidelines for the interpretation of sequence variants: a joint consensus recommendation of the American College of Medical Genetics and Genomics and the Association for Molecular Pathology. Genet Med. 2015;17:405-24.

22. Zhang R, Chen S, Han P, Chen F, Kuang S, Meng Z, et al. Whole exome sequencing identified a homozygous novel variant in CEP290 gene causes Meckel syndrome. J Cell Mol Med. 2020;24:1906-1916.

23. Granata A, Serrano F, Bernard WG, McNamara M, Low L, Sastry P, et al. An iPSC-derived vascular model of Marfan syndrome identifies key mediators of smooth muscle cell death. Nat Genet. 2017;49:97-109.

24. Habashi JP. Losartan, an AT1 antagonist, prevents aortic aneurysm in a mouse model of Marfan syndrome. Science. 2006;312:117-21.

25. Neptune ER, Frischmeyer PA, Arking DE, Myers L, Bunton TE, Gayraud B, et al. Dysregulation of TGF- $\beta$ activation contributes to pathogenesis in Marfan syndrome. Nat Genet. 2003:33:407-11.

26. Regalado ES, Guo DC, Santos-Cortez RLP, Hostetler E, Bensend TA, Pannu $H$, et al. Pathogenic FBN1 variants in familial thoracic aortic aneurysms and dissections. Clin Genet. 2016:89:719-23.

27. Xu S, Li L, Fu Y, Wang X, Sun H, Wang J, et al. Increased frequency of FBN1 frameshift and nonsense mutations in Marfan syndrome patients with aortic dissection. Mol Genet Genom Med. 2020;8:e1041

28. De Cario R, Sticchi E, Lucarini L, Attanasio M, Nistri S, Marcucci R, et al. Role of TGFBR1 and TGFBR2 genetic variants in Marfan syndrome. J Vasc Surg. 2018;68:225-233.e5.

29. Takeda N, Hara H, Fujiwara T, Kanaya T, Maemura S, Komuro I. TGF- $\beta$ signaling-related genes and thoracic aortic aneurysms and dissections. IJMS. 2018:19:2125. 
30. Robertson I, Jensen S, Handford P. TB domain proteins: evolutionary insights into the multifaceted roles of fibrillins and LTBPs. Biochem J. 2011;433:263-76.

31. Chen Y, Dabovic B, Annes JP, Rifkin DB. Latent TGF-beta binding protein-3 (LTBP-3) requires binding to TGF-L for secretion. FEBS Lett. 2002;517:277-80

32. Koli K. Novel non-TGF- $\beta$-binding splice variant of LTBP-4 in human cells and tissues provides means to decrease TGF- $\beta$ deposition. J Cell Sci. 2001,114:2869-78.

33. Miyazono K, Olofsson A, Colosetti P, Heldin C-H. A role of the latent TGF-beta 1-binding protein in the assembly and secretion of TGF-beta 1. EMBO J. 1991;105:1091-101.

34. Zilberberg L, Phoon CKL, Robertson I, Dabovic B, Ramirez F, Rifkin DB. Genetic analysis of the contribution of LTBP-3 to thoracic aneurysm in Marfan syndrome. Proc Natl Acad Sci USA. 2015;112:14012-7.

35. Chen Y, Dabovic B, Colarossi C, Santori FR, Lilic M, Vukmanovic S, et al. Growth retardation as well as spleen and thymus involution in latent TGFbeta binding protein (Ltbp)-3 null mice. J Cell Physiol. 2003;196:319-25.

36. Dabovic B, Levasseur R, Zambuto L, Chen Y, Karsenty G, Rifkin DB. Osteopetrosis-like phenotype in latent TGF- $\beta$ binding protein 3 deficient mice. Bone. 2005;37:25-31.

37. Dabovic B, Chen Y, Colarossi C, Obata H, Zambuto L, Perle MA, et al. Bone abnormalities in latent TGF- $\beta$ binding protein (Ltbp)-3-null mice indicate a role for Ltbp-3 in modulating TGF- $\beta$ bioavailability. J Cell Biol. 2002;156:227-32.

38. Dabovic B, Chen Y, Colarossi C, Zambuto L, Obata H, Rifkin DB. Bone defects in latent TGF-beta binding protein (Ltbp)-3 null mice; a role for Ltbp in TGF-beta presentation. J Endocrinol. 2002;175:129-41.

39. Huckert M, Stoetzel C, Morkmued S, Laugel-Haushalter V, Geoffroy V, Muller J, et al. Mutations in the latent TGF-beta binding protein 3 (LTBP3) gene causebrachyolmia with amelogenesis imperfecta. Hum Mol Genet. 2015;24:3038-49.

40. Noor A, Windpassinger C, Vitcu I, Orlic M, Arshad Rafiq M, Khalid M, et al. Oligodontia Is caused by mutation in LTBP3, the gene encoding latent TGF- $\beta$ Binding protein 3. Am J Hum Genet. 2009;84:519-23.

41. Kaur R, Siddiqui I, Mathur V, Jana M, Kabra M, Gupta N. Bi-allelic loss-offunction novel variants in LTBP3-related skeletal dysplasia: report of first patient from India. Am J Med Genet. 2020;182:1944-6.

\section{Publisher's Note}

Springer Nature remains neutral with regard to jurisdictional claims in published maps and institutionalaffiliations.

Ready to submit your research? Choose BMC and benefit from:

- fast, convenient online submission

- thorough peer review by experienced researchers in your field

- rapid publication on acceptance

- support for research data, including large and complex data types

- gold Open Access which fosters wider collaboration and increased citations

- maximum visibility for your research: over $100 \mathrm{M}$ website views per year

At BMC, research is always in progress.

Learn more biomedcentral.com/submissions 